\title{
New materials for radiation hard semiconductor detectors
}

\author{
P.J. Sellin ${ }^{a}$ and J. Vaitkus ${ }^{b}$ \\ a'Department of Physics, University of Surrey, Guildford UK. \\ ${ }^{\mathrm{b}}$ Institute of Materials Science and Applied Research, Vilnius University, Lithuania
}

\section{On behalf of the RD50 collaboration}

\begin{abstract}
We present a review of the current status of research into new semiconductor materials for use as particle tracking detectors in very high radiation environments. This work is carried out within the framework of the CERN RD50 collaboration, which is investigating detector technologies suitable for operation at the proposed Super-LHC facility (SLHC). Tracking detectors operating at the SLHC in this environment will have to be capable of withstanding radiation levels arising from a luminosity of $10^{35} \mathrm{~cm}^{-2} \mathrm{~s}^{-1}$ which will present severe challenges to current tracking detector technologies. The "new materials" activity within RD50 is investigating the performance of various semiconductor materials that potentially offer radiation hard alternatives to silicon devices. The main contenders in this study are silicon carbide, gallium nitride and amorphous silicon. In this paper we review the current status of these materials, in terms of material quality, commercial availability, charge transport properties, and radiation hardness studies. Whilst these materials currently show considerable promise for use as radiation hard tracking detectors, their ultimate success will depend on the continued improvement of the availability of high quality material.
\end{abstract}

\section{Introduction}

In this paper we review the leading candidates for new materials for radiation hard semiconductor radiation detectors. This review is within the context of the RD50 collaboration, which is developing new semiconductor detectors suitable for use in very high radiation areas in the next generation of high energy physics experiments at the LHC (Moll 2003). The main objective of the RD50 program is to develop detector technologies that can withstand fast hadron fluences of the order of $10^{16} \mathrm{~cm}^{-2}$, with a bunch crossing time as short as $12 \mathrm{~ns}$. These conditions are typical of those proposed for the LHC luminosity upgrade to $10^{35} \mathrm{~cm}^{-2} \mathrm{~s}^{-1}$. This paper summarises the current status of various new materials, which are potential alternatives to silicon for use as radiation hard tracking detectors. We also discuss the developments that are required in terms of both material quality and commercial availability in order for prototype detector devices to be realistically available.

The definition of "new materials" within the RD50 research program covers all possible semiconductor materials that potentially offer high radiation hardness and are suitable as alternatives to silicon for tracking detectors located at very close radii in high luminosity collider experiments. Traditionally silicon has been used almost exclusively for the production of semiconductor tracking detectors, with the availability of very high quality material benefiting from the huge worldwide investment in the silicon microelectronics industry. In the current 
tracking detector program for the LHC, silicon detectors are being utilized in both strip and pixel detector geometries which are expected to survive for ten years of LHC operation. However, the proposed upgrade to the LHC (the Super-LHC or SLHC) will produce a ten times increase in luminosity to approximately $10^{35} \mathrm{~cm}^{-2} \mathrm{~s}^{-1}$. Major efforts are directed within RD50 to carry out defect engineering studies to produce silicon material with improved radiation hardness, suitable for use in SLHC, including studies of oxygenated silicon and other defect engineering techniques. The development of radiation hard silicon detectors has been extensively studied within the RD48 collaboration, see for example (Lindstrom et al. 2001), and more recently within RD50 (Bruzzi 2004; Luukka 2004).

Of the various candidate materials which are potential radiation hard alternatives to silicon, silicon carbide ( $\mathrm{SiC}$ ) is the most mature. This material has been extensively studied over recent years, both as semi-insulating (SI) epitaxial layers and as bulk material. The commercial development of high purity $\mathrm{SiC}$ material has been pioneered by the US company Cree, which produces epitaxial $\mathrm{SiC}$ for high power device applications and bulk $\mathrm{SiC}$ as substrates for InGaN optoelectronic devices. Other new materials which are currently under development both within RD50 and outside are the group III nitrides such as gallium nitride $(\mathrm{GaN})$ which is a wide bandgap material of considerable interest for the fabrication of blue LEDs and lasers, and amorphous materials such as hydrogenated amorphous silicon $(\mathrm{a}-\mathrm{Si}: \mathrm{H})$ and amorphous $\mathrm{SiC}(\mathrm{a}-\mathrm{SiC})$. The new materials activity within RD50 is currently concentrating on a systematic investigation of charge transport, trapping and polarization phenomena in these materials, and the influence of radiation damage on these parameters.

\section{A comparison of potential semiconductors}

A summary of the various significant electrical and mechanical parameters for a range of semiconductor detector materials is shown in Table 1 . These are materials that are either currently used for ionising radiation detection or are potential candidates for future use, including potentially radiation-hard materials such as $\mathrm{GaN}, \mathrm{SiC}$ and amorphous silicon. Also listed are higher-Z compound semiconductors that are of interest for X-ray and gamma ray imaging. In the latter case, the requirement for high- $Z$ materials is primarily driven by the requirement for good detection efficiency at high photon energies. A recent review of some of these materials can be found in (Sellin 2003).

Within the context of RD50, the search for new semiconductor detector materials is concentrated on their ability to operate at extremely high radiation fields, such as a fast hadron flux greater than $1 \times 10^{16} \mathrm{~cm}^{-2}$. Besides the radiation damage effects to the detector material caused by this level of primary radiation, there is the additional consideration of material activation at high fluence. Material activation rules out the use of those elements which readily undergo nuclear reactions with hadrons, which includes aluminium and most of the heavy elements.

The degradation of detector performance by radiation damage can occur through a variety of interrelated processes. Whilst generally caused by the creation of charge trapping centres in the semiconductor, it is important to identify the various processes and their relative importance in any particular material. These process include (a) a reduction in charge drift length, and hence signal amplitude, due to a decrease in either the electron or hole mobility-lifetime product, (b) the increase of carrier drift time due to reduced mobility, such that the induced signal is slower or 
comparable to the shaping time of the readout electronics, causing a 'ballistic deficit', (c) decreasing penetration of the electric field in the detector due to an increase in carrier concentration, causing a reduction in the width of the space charge region, and (d) the formation of additional space charge in the device due to deep centers which can cause polarisation phenomena.

The precise balance of the effects observed in a radiation damaged detector is complex, and specific to each material system and device geometry. In general the most serious radiationinduced effects are the reduction in either mobility or carrier lifetime due to the introduction of defects, plus the reduction of the space charge region thickness, both leading to loss of signal amplitude. In highly irradiated silicon the reduction in thickness of the space charge region leads ultimately to type inversion, in which the n-type bulk silicon becomes p-type. In an irradiated detector increasing the bias voltage can generally recover some lost signal amplitude by reducing the drift time of the carriers through the device. However this is only practicable if the detector leakage current remains at a reasonable level, since increasing the bias voltage will ultimately cause an unacceptable signal/noise ratio, or even device breakdown.

Of the materials listed in Table 1, semiconductors such as the Group II tellurides CdTe and $\mathrm{CdZnTe}$, and other higher-Z materials such as $\mathrm{HgI}_{2}$ and $\mathrm{TlBr}$ can be discounted as tracking detectors due to their readiness to undergo hadronic reactions and their long radiation length. The focus for detector development with these materials remains strongly for X-ray and gamma ray imaging applications. It is possible that these materials may be useful for other applications in LHC experiments, but not within the scope of the RD50 collaboration. Diamond represents a special category of material which, although potentially suitable as a very radiation hard detector, remains limited by poor supply and high cost. The development and current status of polycrystalline diamond tracking detectors has been extensively reported by the RD42 collaboration (see recent reviews by (Mainwood 2000; Adam et al. 2003)) and is outside the scope of this paper.

$\mathrm{SiC}$ is currently the most promising alternative material to silicon for use in future radiation hard detector devices, and a more detailed description of the current status of $\mathrm{SiC}$ is presented in the following section. Preliminary investigations have demonstrated good radiation hardness in bulk $\mathrm{SiC}$ detectors but polarization effects and the presence of crystalline defects (such as micropipes and dislocations) limit the charge transport performance of bulk material. Improvements in epitaxial $\mathrm{SiC}$ growth means that semi-insulating epitaxial $\mathrm{SiC}$ layers have recently become available, with thicknesses of up to $50 \mu \mathrm{m}$. However the supply of high quality epitaxial SiC, which is currently driven by electronic devices and LED applications, is still restricted to Cree in the US and a handful of other minor suppliers. There is currently no commercial source of epitaxial SiC of the thickness normally required for detector applications, eg. 100-300 $\mu \mathrm{m}$.

Another interesting material is $\mathrm{GaN}$, which has recently seen significant developments for use in light emitting devices and UV photosensors. The semiconductor parameters and charge transport properties of $\mathrm{GaN}$ are also well suited for applications in high frequency and high voltage electronics. Advances in GaN growth technology has led to the development of epitaxial growth of GaN layers, heterostructures and nanocrystallites. The main limitation of this material technology is related to problems with the substrate material required for epitaxial growth, typically sapphire. Misfit of the lattice constant between the epitaxial layer and the substrate creates a large density of dislocation defects in the GaN, particularly at the interface between the epitaxial material and the 
substrate. For nanostructure based LEDs this defect density is not necessarily a problem, however in thick epitaxial material of the type required for radiation detectors extended defects such as microcracks limit device quality. However rapid advances in $\mathrm{GaN}$ material quality are currently being reported, with the prospect of free-standing GaN wafers already becoming commercially available.

In comparison to other candidate materials, $\mathrm{GaN}$ is of particular interest for use as a radiation hard ionizing radiation detector due to the high ionic bond strength and large crystal density. These properties suggest that $\mathrm{GaN}$ may exhibit a greater radiation hardness than other comparable compound semiconductors and will suffer less from the influence of interstitial impurities. According to the presently known data, the displacement energy for GaN is not so high but these reported measurements were performed on material with a high density of defects. The first investigations of epitaxial GaN as an ionizing radiation detector were performed within the RD50 collaboration and the results are promising (Vaitkus et al. 2003; Sellin et al. 2004).

An alternative gallium-based semiconductor, GaAs, was previously investigated for use as a possible radiation hard detector material by the CERN RD8 collaboration (see for example (Bates et al. 1998)). These studies, which concentrated on bulk SI GaAs, were not successful due mainly to short carrier lifetimes ( $<10 \mathrm{~ns}$ ) caused by high levels of the native EL2 deep defect. Significant improvements have recently been made in the performance of epitaxial GaAs X-ray detectors with near Fano-limited performance reported, for example achieving a noise of $242 \mathrm{eV} \mathrm{FWHM}$ at $20^{\circ} \mathrm{C}$ (Owens et al. 1999; Bertuccio et al. 2003). However further development of epitaxial GaAs X-ray detectors is seriously limited by the lack of availability of high purity thick epitaxial material. The development of bulk GaAs using defect engineering techniques has attracted some attention, for example in the same way as in a-Si by creating a large concentration of defects due to deep centres such as chromium. Recent results report good charge transport performance in such highlycompensated GaAs material (Aizenshtadt et al. 2000) and future developments in highly-doped GaAs may produce devices with performance suitable for RD50 activities.

\section{Silicon Carbide}

\subsection{SiC materials properties}

The growth of high resistivity $\mathrm{SiC}$ for radiation detector applications can be achieved using two different techniques, either as bulk material grown as a single crystal, or as an epitaxial layer. Bulk $\mathrm{SiC}$ is currently the only route to produce thick wafers (eg. $100-500 \mu \mathrm{m}$ thickness) however the quality of this material is relatively poor. Conversely epitaxial $\mathrm{SiC}$ grown onto a substrate wafer can produce high purity material but with a thickness that is currently limited to approximately 50 $\mu \mathrm{m}$. At the present time therefore neither type of material is ideally suited for detector use. However both growth methods are undergoing rapid developments and the quality of SiC material continues to improve. It is likely that thick epitaxial $\mathrm{SiC}$ will provide the best source of detector grade material, provided that a fast and hence cost effective epitaxial growth method can be commercially developed. 
$\mathrm{SiC}$ offers excellent electrical properties that are well-suited for radiation detector applications, including a high breakdown electric field, good mobilities, and a high saturated electron drift velocity. A summary of the main properties of $4 \mathrm{H}$ and $6 \mathrm{H} \mathrm{SiC}$ has previously been given in Table 1. The high bandgap energy of $\mathrm{SiC}$ produces extremely low dark currents, and allows operation at high temperature, eg. up to $700^{\circ} \mathrm{C}$. The significantly higher electron mobility observed in $4 \mathrm{H} \mathrm{SiC}$ makes this the preferred polytype for radiation detector applications. SiC has a high displacement threshold energy of $21.8 \mathrm{eV}$, which should lead to a high radiation hardness. Consequently the material is of particular interest for detector operation in a high radiation environment, such as nuclear plant monitoring and as an inner tracker in future particle physics experiments.

The supply of bulk $\mathrm{SiC}$ is driven by the use of this material as a high quality substrate for $\mathrm{SiC}$ and $\mathrm{GaN}$ microelectronics. To achieve high resistivity SI SiC, vanadium is incorporated into the crystal during growth, acting as an amphoteric deep dopant. Various growth techniques have been developed to produce large-area single crystal $\mathrm{SiC}$, including the modified Lely method (Bickermann et al. 2001) and by physical vapour transport (Barrett et al. 1993; Hobgood et al. 1994; Augustine et al. 1997). The main commercial growth technique for SiC substrates is physical vapour transport, with material available from Cree and a number of other suppliers (eg. SiXON, Sterling, Nippon Steel). Okmetic has recently developed an alternative high temperature CVD method for substrate growth, producing high quality SI material. Commercial SI SiC wafers are mainly available in the $4 \mathrm{H}$ polytype, with Cree as the dominant supplier offering $\mathrm{SI} 4 \mathrm{H}-\mathrm{SiC}$ wafers up to 3 inches in diameter. SI SiC wafers tend to show poor homogeneity, particularly due to non-uniform incorporation of vanadium and radial variations in impurity distributions. Contactless resistivity measurements show significant variations, particularly towards the edges of SI wafers, possibly due to the presence of vanadium-rich precipitates (Bickermann et al. 2001). Alternative resistivity measurements using removable graphite contacts have also be used as a rapid method of assessing SI SiC wafer quality (Muzykov et al. 2003).

A variety of suppliers offer epitaxial SiC material, with Cree being the dominant supplier. The SI epitaxial material is lightly n-type and has a typical carrier concentration in the range $5 \times 10^{14}-5 \mathrm{x}$ $10^{15} \mathrm{~cm}^{-3}$. Currently SI epitaxial wafers are available with a thickness of up to $50 \mu \mathrm{m}$. The epitaxial layer is grown on a low resistivity n-type $4 \mathrm{H}-\mathrm{SiC}$ substrate, on top of a $1 \mu \mathrm{m}$ thick buffer layer. The defect density in this type of thick SI epitaxial material can be as low as $\sim 30 \mathrm{~cm}^{-2}$. The availability of thicker epitaxial SI material with ultra-low defect density is key to the successful development of $\mathrm{SiC}$ radiation detectors, with the possibility of free-standing thick epitaxial layers in the future. Some alternative sources of epitaxial $\mathrm{SiC}$ exist, limited mainly to research laboratories. The use of a vertical hot-wall chemical vapor deposition method has been reported (Fujihira et al. 2003), capable of producing $\mathrm{SiC}$ at high growth rates. 4H-SiC epilayers, with a quality superior to conventional material, were grown at $25-60 \mu \mathrm{m} / \mathrm{h}$, which is a growth rate $5-10$ times higher than conventional methods.

A large number of studies have been reported of the amphoteric dopant properties of vanadium in $\mathrm{SiC}$, and a wide range of deep levels have been identified. When acting as a deep acceptor, vanadium forms a level at an energy $0.8-1.0 \mathrm{eV}$ below the conduction band, with the exact energy depending on the polytype (Jenny et al. 1996), and compensates the shallow donors introduced by impurities from the growth process (Schneider et al. 1990). The dominant vanadium donor state has been attributed to a level at $1.6-1.7 \mathrm{eV}$ below the conduction band (Mitchel et al. 
1999). Many authors report a range of deep levels in conducting SiC, identified using deep level transient spectroscopy (DLTS) and optical admittance spectroscopy. A recent review by Dalibor (Dalibor et al. 1997) presents DLTS investigations of deep defects in $3 \mathrm{H}, 4 \mathrm{H}$ and $6 \mathrm{H} \mathrm{SiC}$ polytypes. DLTS studies of deep level activation energies and concentrations in SI epitaxial $4 \mathrm{H}-$ SiC Schottky detectors have also be reported in (Castaldini et al. 2002).

The use of bulk $\mathrm{SiC}$ for microelectronics and detector applications continues to be limited by a high dislocation density, consisting of inclusions, voids and micropipes. Micropipes are hollow defects that extend deep into the material, with radii varying from tens of nanometres to tens of microns. Micropipes can affect the performance SiC devices by acting as centers of breakdown at high electric field strength due to the formation of plasma, or alternatively acting as trapping centers. The exact formation mechanism of micropipes is still unclear, however these defects are often associated with a screw dislocation. The spiral growth mechanism of micropipes has been investigated using AFM studies in 6H-SiC (Giocondi et al. 1997). The density of micropipes is typically in the range $50-1000 \mathrm{~cm}^{-2}$, which tends to increase in large diameter crystals. Commercially grown conducting $\mathrm{SiC}$ wafers can be obtained with micropipe densities less than 20 $\mathrm{cm}^{-2}$, and it is expected that similarly low densities will soon be achieved for SI bulk material.

Contactless assessment of defect densities in $\mathrm{SiC}$ can be carried out using a variety of techniques, including X-ray phase imaging, transmission electron microscopy, and optical imaging. X-ray phase imaging uses a highly coherent beam of synchrotron photons, and is particularly sensitive to variations in electron density, even in low-Z materials (Milita et al. 1999). For relatively thin samples which are optically transparent, polarised optical microscopy is a useful low-cost method which images stress birefringence in the vicinity of micropipes and dislocations. This has been applied to $4 \mathrm{H}$ and $6 \mathrm{H}$ material grown by the modified Lely method (Ma et al. 2002) which has shown relatively high defect densities $\left(10^{4}-10^{5} \mathrm{~cm}^{-2}\right)$.

For epitaxial $\mathrm{SiC}$ various measurements of Hall mobility have been reported, as a function of carrier concentration. High quality epitaxial n-type $6 \mathrm{H}-\mathrm{SiC}$ shows electron Hall mobility values of $\sim 200-300 \mathrm{~cm}^{2} / \mathrm{Vs}$ at room temperature, rising to $10^{3}-10^{4} \mathrm{~cm}^{2} / \mathrm{Vs}$ at $50 \mathrm{~K}$ (Dhar et al. 2000). In ptype $\mathrm{SiC}$ the hole mobility is typically around $50 \mathrm{~cm}^{2} / \mathrm{Vs}$ at room temperature. In contrast there is very little data available for carrier mobilities in bulk material. As a consequence of the hexagonal structure of $\mathrm{SiC}$, there is a significant anisotropy in the electron mobility between motion of carriers parallel and perpendicular to the $c$-axis. Anisotropy measurements made on $4 \mathrm{H}$ and $6 \mathrm{H}$ single crystal $\mathrm{SiC}$ show quite different behaviour, with the parallel Hall mobility in $4 \mathrm{H}$ material higher than the perpendicular mobility, whereas the opposite case was observed in $6 \mathrm{H}$ (Schadt et al. 1994).

Migration of defects induced by carrier recombination is an important feature in irradiated SiC, which has been observed, for example, in the recombination-induced transform of stacking faults (Galeckas et al. 2002; Jacobson et al. 2004). Such effects are potentially dangerous in highly irradiated materials and can lead to a decrease in radiation hardness. Defect engineering techniques to improve the material properties of compound semiconductors have been proposed for GaAs by the use of isovalent doping (see for example (Holmes et al. 1988; Saravanan et al. 2000)). Similar approaches could be used for $\mathrm{SiC}$ growth to investigate possible improvements in radiation hardness, for example by adding a small concentration of Ge. An attempt has been made in this direction by the healing of micropipes in $\mathrm{SiC}$ wafers (Filip et al. 2004). 


\subsection{Typical device performance}

The first measurements of $\mathrm{SiC}$ radiation detectors were carried out more than 40 years ago to assess their use as neutron detectors within the nuclear power industry. SiC diode detectors produced a good alpha particle response, even at temperatures of $700^{\circ} \mathrm{C}$. Various workers have reported results of thermal neutron measurements using $\mathrm{SiC}$ detectors in which the neutron sensitivity has been achieved with either a ${ }^{235} \mathrm{U}$ conversion film, or by beryllium implantation. However the performance of these early devices was generally limited by defects and impurities in the material and further development was prevented by the lack of availability of high quality SiC.

More recently the development of $\mathrm{SiC}$ radiation detectors has seen renewed interest due to the development of high purity bulk and epitaxial SiC material. One of the first investigations of the alpha particle detection performance of $4 \mathrm{H}$ epitaxial $\mathrm{SiC}$ was by Ruddy et al (Ruddy et al. 1998), using high quality epitaxial layers that were $4-8 \mu \mathrm{m}$ thick and lightly-doped with nitrogen at $10^{15}$ $\mathrm{cm}^{-3}$. Full depletion of the $8 ? \mu \mathrm{m}$ thick device was achieved at a bias of $60 \mathrm{~V}$, and this structure showed good sensitivity to alpha particles. Two types of diode structures were fabricated, using nickel Schottky contacts, and with a $\mathrm{p}^{+}-\mathrm{n}$ contact. Slightly better alpha particle resolution observed from the Schottky device and no significant change in the alpha particle response was observed when the detectors were operated at $90^{\circ} \mathrm{C}$.

Similar alpha particle and MIP response measurements have been made on devices fabricated on 4H SiC epitaxial layers by Alenia (Nava et al. 1999; Verzellesi et al. 2002; Bruzzi et al. 2003). In these devices the epitaxial layer was up to $50 \mu \mathrm{m}$ thick, with a nominal n-type nitrogen doping of $9 \times 10^{14}-2 \times 10^{15} \mathrm{~cm}^{-3}$, and fabricated using a gold Schottky contact. Evidence from capacitancevoltage measurements showed a variation in doping concentration as a function of depth through the epitaxial layer, with a minimum value of $5 \times 10^{14} \mathrm{~cm}^{-3}$ close to the Schottky contact. A maximum depletion thickness of $21 \mu \mathrm{m}$ was achieved, at a bias voltage of approximately $400 \mathrm{~V}$. The measured charge collection efficiency (CCE) for $5.49 \mathrm{MeV}$ and $2.00 \mathrm{MeV}$ alpha particles was $100 \%$ and no priming or polarisation effects were observed up to bias voltages greater than $500 \mathrm{~V}$, confirming the absence of significant trap concentrations in this material. Ivanov et al (Ivanov et al. 2005) report similar alpha particle spectra, with sufficient energy resolution to resolve alpha peak sub-structure from ${ }^{241} \mathrm{Am}$ (Figure 1). The successful operation of SiC devices as alpha particle detectors at zero applied bias indicates a substantial charge signal can be obtained from minority carrier diffusion in $\mathrm{SiC}$, with measured hole diffusion lengths of $\sim 11 \mu \mathrm{m}$ (Nava et al. 2003; Ivanov et al. 2005).

The response to MIPs of devices fabricated from ultra-low defect concentration material has been reported (Nava et al. 2004), using $40 \mu \mathrm{m}$ thick 4H-SiC grown by the Institute of Crystal Growth, Berlin. These devices showed a net doping concentration of $6 \times 10^{13} \mathrm{~cm}^{-3}$, and full depletion at $60 \mathrm{~V}$. MIP pulse height spectra acquired using a ${ }^{90} \mathrm{Sr}$ source (Figure 2) show the landau distribution clearly separated from the Gaussian noise pedestal at high bias voltage, with a peak centroid of 2200 electrons $(\mathrm{SNR} \sim 6)$ at $60 \mathrm{~V}$.

The characterisation of $\mathrm{SiC}$ radiation detectors for other applications has also been reported, including low energy X-ray spectroscopy and radiation dosimetry. For low energy $(<20 \mathrm{keV}) \mathrm{X}$ - 
ray detection, $\mathrm{SiC}$ potentially offers higher spectroscopic performance than silicon for high temperature measurements, due to the lower reverse bias current density (Bertuccio et al. 2003; Bertuccio et al. 2004). Measurements of a gold contact $4 \mathrm{H} \mathrm{SiC} \mathrm{Schottky} \mathrm{diode} \mathrm{at} 340 \mathrm{~K}$ show a current density of $17 \mathrm{pA} / \mathrm{cm}^{2}$, more than two orders of magnitude lower than commercial silicon devices. Good spectroscopic performance of has been demonstrated for the low energy photon lines from ${ }^{241} \mathrm{Am}$, both at room temperature (Figure 3) and at $100^{\circ} \mathrm{C}$.

The dosimetric response of epitaxial 4H SiC detectors has been reported in (Bruzzi et al. 2001), which shows good linearity as a function of absorbed dose and a sensitivity comparable to that of a commercial ionisation chamber. Using an unbiased $30 \mu \mathrm{m}$ thick SiC Schottky diode, a sensitivity of $14.1 \mathrm{nC} / \mathrm{Gy}$ was obtained for ${ }^{60} \mathrm{Co}$ dose up to $1 \mathrm{~Gy}$. The performance of $\mathrm{SiC}$ dosimeters is comparable to that of Si photodiodes, and potentially offer greater resistance to radiation damage. However further studies are required to investigate the tissue equivalence correction factors required for $\mathrm{SiC}$ dosimetry.

In comparison with epitaxial $\mathrm{SiC}$, much less data has been published concerning the performance of devices fabricated from bulk material. Potentially bulk $\mathrm{SiC}$ is an easier route for the production of thicker devices, however the relatively poor charge transport characteristics compared to epitaxial SiC severely limits device performance. Systematic studies are still required to investigate the correlation between dopant concentration and mobility-lifetime products in SI material.

Some of the first measurements on bulk $\mathrm{SiC}$ detectors were of the alpha particle response of devices fabricated from $310 \mu \mathrm{m}$ thick SI $4 \mathrm{H}-\mathrm{SiC}$, grown by Cree (Rogalla et al. 1999). Ohmic contacts were applied using aluminium, and the measured resistivity of the material was $5 \times 10^{10}$ $\Omega \mathrm{cm}$. Pulse height spectra acquired using minimum ionising particles (MIPs) from ${ }^{90} \mathrm{Sr}$ showed a Landau distribution resolved from the noise, with a most probably signal amplitude of 1950 electrons at a bias of $500 \mathrm{~V}$. The maximum CCE observed in this device was $12 \%$, limited by trapping from deep levels in the material.

Detectors fabricated from SI 6H-SiC, grown by the modified Lely method, were reported by Bruzzi et al (Bruzzi et al. 2001). The devices were 250-300 $\mu \mathrm{m}$ thick, fabricated with $100 \mathrm{~nm}$ thick aluminium contacts. Current-voltage measurements up to $800 \mathrm{~V}$ at room temperature showed ohmic behaviour, with a bulk resistivity of $10^{11} \Omega \mathrm{cm}$. Charge sensitivity measurements were carried out using a high intensity beam of $22 \mathrm{MeV}$ electrons with a sensitivity of approximately 16 $\mathrm{nC} / \mathrm{Gy}$, although no pulse-counting data were presented.

Alpha particle spectra were reported from devices fabricated from bulk $6 \mathrm{H} \mathrm{SiC}$, using $100 \mu \mathrm{m}$ thick SI material produced by Cree (Cunningham et al. 2003). A $100 \mathrm{~nm}$ thick nickel top contact was applied by electron beam evaporation, and alpha particle spectra were measured with a maximum CCE of $60 \%$ at $600 \mathrm{~V}$ bias.

\subsection{Radiation Damage}

For potential applications in particle physics experiments, the resistance of SiC detectors to radiation damage, particularly from high energy hadrons, is crucial. Various measurements of radiation damage from charged particles and $\mathrm{MeV}$ photons have been reported, and studies have also been made of the radiation hardness of SiC UV photodetectors, as recently reviewed in 
(Lebedev et al. 2004). Radiation hardness tests of a commercial SiC UV photodiode supplied by Cree were reported for ${ }^{60} \mathrm{Co}$ gamma, proton and neutron irradiation (Metzger et al. 2002). Irradiation tests with intense ${ }^{60} \mathrm{Co}$ sources showed, for a total dose of $980 \mathrm{kGy}$, a $10 \%$ drop in gamma signal amplitude and a small increase in the device dark current. Irradiation with $32 \mathrm{MeV}$ protons up to a fluence of $8.5 \times 10^{12} \mathrm{~cm}^{-2}$ showed no loss of signal amplitude, whilst irradiation with $14 \mathrm{MeV}$ neutrons showed no loss of signal amplitude up to $2 \times 10^{12} \mathrm{~cm}^{-2}$, then a $20 \%$ drop in amplitude up to $4 \times 10^{12} \mathrm{~cm}^{-2}$. Photoluminescence studies of $4 \mathrm{H}-\mathrm{SiC}$ irradiated with heavy ions (eg. $1 \times 10^{9} \mathrm{~cm}^{-2}$ of $710 \mathrm{MeV} \mathrm{Bi}$ ) demonstrate the formation of radiation-induced defects similar to those created by neutron irradiation, with the defect creation mechanisms dominated by elastic collisions (Kalinina et al. 2004). The radiation resistance of 4H-SiC Schottky diode detectors to thermal neutrons has also been demonstrated (Seshadri et al. 1999) with a significant reduction in charge collection efficiency observed only at neutron fluences $>5 \times 10^{16} \mathrm{~cm}^{-2}$ (Figure 4). Similar radiation hardness measurements of $4 \mathrm{H}-\mathrm{SiC}$ detectors to $1 \mathrm{MeV}$ fast neutrons and protons have also been reported (Sciortino et al. 2005). In this data the alpha particle CCE for a $21 \mu \mathrm{m}$ thick $4 \mathrm{H}-\mathrm{SiC}$ detector showed a significant decrease at neutron fluences above $1 \times 10^{14} \mathrm{~cm}^{2}$ (Figure 6), reducing to a CCE of $\sim 10 \%$ at a neutron fluence of $1 \times 10^{17} \mathrm{~cm}^{2}$.

Similar results have been reported for radiation hardness measurements of bulk SiC detectors. The radiation hardness of vanadium-doped SI bulk $4 \mathrm{H}-\mathrm{SiC}$ was investigated at pion fluences in excess of $10^{13} \mathrm{~cm}^{-2}$ (Rogalla et al. 1999).. Well resolved alpha particle spectra were observed after irradiation, even though charge drift lengths were limited in these devices. In a second study of $100 \mu \mathrm{m}$ thick $6 \mathrm{H} \mathrm{SiC}$ detectors charge collection efficiencies of approximately $50 \%$ were measured after irradiation with $10^{13}$ pions $/ \mathrm{cm}^{2}$ (Figure 5) (Cunningham et al. 2003).

At the present time, the emphasis in $\mathrm{SiC}$ radiation detectors has moved away from bulk material, and this is unlikely to change unless a significant improvement occurs in the growth of higher quality bulk SiC. The recent improvements in reducing defect concentrations in epitaxial SiC means that this material is likely to dominate over bulk $\mathrm{SiC}$ for the foreseeable future, and further improvements in the supply and quality of epitaxial material are expected.

\section{Gallium Nitride}

Rapid progress of epitaxial growth techniques for $\mathrm{GaN}$ material makes this material attractive for applications in high-temperature/high-power electronic devices operating at high frequencies as well as in blue-UV optoelectronic devices (Nakamura et al. 2000; Shur et al. 2004). Semiinsulating $\mathrm{GaN}$ is a promising material for the detection of $\mathrm{UV}$ radiation but also due to its high density, stability and high threshold voltage has considerable potential for use in ionising radiation detectors.

The availability of SI GaN material of sufficient quality for detector use is currently restricted to thin epitaxial layers, typically grown on a sapphire substrate. The development of new growth technologies capable of producing $\mathrm{GaN}$ material with low dislocation densities is currently ongoing, with a buffer layer commonly used between the sapphire and the GaN to reduce the GaN dislocation density to $10^{6} \mathrm{~cm}^{-2}$ or less. The use of low dislocation density GaN for UV photo detectors has recently been reported (Pau et al. 2004), and the availability of thick layers of high quality material continues to develop. Studies of GaN epitaxial samples grown by MOCVD with different levels of compensation on sapphire substrates were recently reported (Wang et al. 2000). 
The compensation properties and resistivity of these layers were changed by variation of the substrate temperature and the trimethyl gallium flow rate during growth, without the use of intentional doping. This material produced SI epitaxial GaN that was $2-2.5 \mu \mathrm{m}$ thick, with a carrier concentration of $\sim 3 \times 10^{16} \mathrm{~cm}^{-3}$.

First measurements have been made of $\mathrm{GaN}$ radiation detector test structures, fabricated from thin (typically $2 \mathrm{~mm}$ ) semi insulating epi layers grown on sapphire substrates. The response of Schottky pad detectors to alpha particles and protons has been reported (Vaitkus et al. 2003; Sellin et al. 2004) which have demonstrated CCE values close to $100 \%$ (Figure 7). The temporal dependence of dark current and photo-conductivity transients has also been studied (Vaitkus et al. 2003), showing a wide range of time constants observed in transient analysis. These thin detector structures have established the baseline performance of $\mathrm{GaN}$ particle detectors and provide useful measurements of the charge transport properties and radiation hardness of $\mathrm{GaN}$.

Little radiation hardness data is currently available for $\mathrm{GaN}$, which remains a topic of interest to both the radiation detector and optoelectronic communities. Studies of excitonic luminescence of GaN after irradiation with $0.42 \mathrm{MeV}$ electrons established that damage was generated exclusively in the nitrogen sub lattice (Yang et al. 2003). No displacement of the nitrogen atom was observed, which suggests that the nitrogen sub lattice repairs itself through room temperature annealing. Similar studies on GaN LEDs using $1400 \mathrm{keV}$ electrons measured a displacement energy for the gallium atom of $19.2 \mathrm{eV}$, which is significantly higher than for GaAs, and comparable to silicon carbide (Ionascut-Nedelcescu et al. 2002). Photoluminescence and carrier lifetime studies have investigated the role of radiation damage in both MOCVD-grown epilayers and HVPE-grown free standing material (500 $\mu \mathrm{m}$ thickness) (Gaubas et al. 2005). Clear evidence is observed for the introduction of non-radiative defects at high neutron fluences that quench the PL bands in this material. However the inter-play between as-grown material disorder and radiation induced defects still requires further studies.

For thin radiation pad detectors no significant reduction in CCE is observed after irradiation to a dose of $600 \mathrm{Mrad}$ with $10 \mathrm{keV}$ X-rays (Rahman et al. 2003). However recent first data from a single thin $\mathrm{GaN}$ device indicates a significant reduction in alpha particle CCE for neutron fluences in excess of $1 \times 10^{14} \mathrm{~cm}^{-2}$ (Vaitkus et al. 2003). As shown in Figure 8, the CCE reduced dramatically from $80 \%$ at $5 \times 10^{14} \mathrm{~cm}^{-2}$ to less than $10 \%$ at $1 \times 10^{15} \mathrm{~cm}^{-2}$. The decrease in CCE for proton irradiated SI GaN was not so significant, and equal to $14 \%$ at $1 \times 10^{16} \mathrm{~cm}^{-2}$ (Vaitkus et al. 2005). However further systematic studies are required to confirm these initial results.

The main challenge for $\mathrm{GaN}$ is the availability of high quality thick SI GaN layers. Currently SI epitaxial layers are only commercially available with thicknesses of less than $10 \mu \mathrm{m}$. A early growth technique for single crystal GaN was that of high-pressure high-temperature growth, pioneered in Warsaw (Porowski et al. 1997). However this method is only capable of producing platelets approximately $1 \mathrm{~cm}$ in diameter. More recently there have been rapid advances in epitaxybased GaN growth, and free-standing conducting wafers of $\mathrm{GaN}$ are now available grown by hydride vapour phase epitaxy (HVPE). The HVPE method can achieve growth rates of up to 150 $\mu \mathrm{m} / \mathrm{hr}$ with laser-assisted separation of the film from the sapphire substrate. There are many reports of the growth and characterisation of free standing conducting $\mathrm{GaN}$ wafers grown by HVPE, see for example references (Kelly et al. 1999; Freitas et al. 2002; Xu et al. 2002). 
Commercial suppliers such as Lumilog are currently offering $400 \mu \mathrm{m}$ thick 2 " wafers of n-type free standing $\mathrm{GaN}$, and similar SI free standing wafers will be available very shortly. Consequently $\mathrm{GaN}$ has a promising future for use in radiation detectors, and further progress in this area will rely on the continuing improvements in material growth and a reduction in material cost. However the radiation hard nature of $\mathrm{GaN}$ detectors for use in neutron fluences above $1 \times 10^{14} \mathrm{~cm}^{-2}$ and the origin of the low breakdown field in epitaxial SI-GaN need urgent further investigation, including the performance of MOCVD and HVPE-grown thick film material.

\section{Hydrogenated amorphous silicon}

During the last 20 years great progress has been made in the use of hydrogenated amorphous silicon (a-Si:H) for various sensors and high resolution imaging devices including copying machines, solar cells and panel displays. The optical bandgap in pure a-Si is $1.7 \mathrm{eV}$, and it was observed up to $1.9 \mathrm{eV}$ for a-Si:H (Alvarez et al. 1995). The density is quite similar to crystalline silicon at approximately $2.3 \mathrm{~g} / \mathrm{cm}^{3}$ for a-Si:H(8\%) and $2.1 \mathrm{~g} / \mathrm{cm}^{3}$ for a-Si:H(15\%) (Schmidt et al. 1995). Since a-Si:H films can be deposited at low cost onto very large area substrates, it has been an appealing material for photovoltaic solar energy conversion. Large area X-ray imaging devices have also been developed, see for example (Street et al. 1996), using a-Si TFT arrays coupled to a scintillator or phosphor screen. Most recently there have been several reports of the investigation of a-Si for direct radiation detection in high-energy physics.

Studies have been made of the possible improvement in radiation hardness of amorphous silicon, demonstrated in a-Si:H, in comparison to crystalline silicon (c-Si). Photoconductivity spectra and radiation-induced conductivity have been measured in situ, under $17 \mathrm{MeV}$ proton irradiation, as a simulation of the radiation environment typical of a fusion experiment. In c-Si, significant deterioration of the optoelectronic properties was observed, caused by lattice damage due to radiation-induced defects. On the contrary, a-Si:H showed remarkable radiation resistance against proton bombardment. The stable photoconductivity in a-Si:H was accompanied by enhanced structural metastability under irradiation. The good radiation resistance is also due to the characteristics of high-energy protons, i.e., electronic excitation dominant over structural displacement damage. The mechanism was ascribed to spontaneous recombination of displaced atoms, promoted by electronic excitation (Kishimoto et al. 1998).

The main problem limiting the development of a-Si:H radiation detectors is the technical difficulty of depositing very thick a-Si:H layers. In this context $50 \mu \mathrm{m}$ is considered the minimum thickness required to produce a charge signal of at least 4000 electron-hole pairs per MIP. Recently such layers have been successfully grown and their charge collection efficiency demonstrated (Juska et al. 1995) In the past, the readout of such a low signal level turned out to be the major obstacle for radiation tracking detectors. Recently, a radiation sensor for charged particles based on the vertical integration design has been proposed (Anelli et al. 2004). In this proposed device direct a-Si:H deposition onto a suitable readout circuit is achieved by a plasma enhanced chemical vapour deposition (PE-CVD) process. The deposition is performed at a relatively low temperature, 200 $250^{\circ} \mathrm{C}$, which is compatible with post processing on CMOS electronic wafers. The PE-CVD deposition of a-Si:H for use as a radiation detector requires similar deposition technology as used for the manufacturing of a-Si:H photovoltaic solar panels, except for an increased thickness and a 
reduction in the dangling bond density to no more than $10^{15} \mathrm{~cm}^{-3}$. Test structures for a-Si:H singleparticle detection have been developed using n-i-p photodiodes (Figure 9), which showed a low dark current of $\sim 6 \mathrm{pA} / \mathrm{cm}^{2}$ for mean field strengths below $1 \mathrm{~V} / \mu \mathrm{m}$ (Despeisse et al. 2004). These devices showed a prompt electron drift time of $\sim 4 \mathrm{~ns}$, with a slower component due to hole transport. The high integration level of the detecting device and readout electronics produces a significant improvement in signal-noise ratio for MIPs, and a considerable reduction in device fabrication costs. However the measured signal amplitude from a-Si:H is still severely restricted due to the very short hole carrier drift length observed in this material.

The opto-electronic properties of a-Si:H is dependent on the as-grown hydrogen concentration. At low hydrogen concentration randomly distributed monohydride groups are observed, whilst at higher concentration $(>20 \% \mathrm{H}$ ) hydrogen nanoclusters on a scale of less than $2 \mathrm{~nm}$ were observed (Reimer et al. 1988). Such clusters can exhibit either sp2 or sp3 type bonding, and significantly effect the material properties. Defects related to hydrogen exhibit a creation activation energy of approximately $0.92 \mathrm{eV}$ which corresponds to a process time constant (at $300 \mathrm{~K}$ ) of $10^{6} \mathrm{~s}$. Conversely the defect annealing activation energy is approximately $1.27 \mathrm{eV}$ which corresponds to process time constant (at $300 \mathrm{~K}$ ) of $10^{8} \mathrm{~s}$ (Jackson et al. 1988). Fluorine can also be incorporated into a-Si in a variety of different forms such as dispersed fluorine, clustered fluorine, $\mathrm{SiF}_{4}$ molecules and $\mathrm{SiF}_{3}$ species.

Metastable defects can be formed in a-Si by ionizing irradiation, such as low energy electrons and $\mathrm{X}$ - and gamma rays. At higher energies (typically $1-20 \mathrm{MeV}$ ) radiation-induced defects related to $\mathrm{Si}$ displacement are also created. These defects, which include silicon interstitials, can be subsequently annealed, for example after $12 \mathrm{~h}$ at room temperature. The defects generated in a-Si can be produced by the excitation of metastable dangling bonds and by charge trapping in $\mathrm{Si}-\mathrm{Si}$ weak bonds, and the stabilisation of such defects can be strongly influenced by the presence of hydrogen (Schneider et al. 1988).

The mu-tau product $(\mu \mathrm{t})$ for undoped a-Si:H is $\sim 10^{-7} \mathrm{~cm}^{2} / \mathrm{V}$ for electrons and $\sim 10^{-8} \mathrm{~cm}^{2} / \mathrm{V}$ for holes (Wyrsch et al. 1995). At high electric field the electron mobility saturates and becomes equal to $2.5 \mathrm{~cm}^{2} / \mathrm{Vs}$ at $0.5 \mathrm{MV} / \mathrm{cm}$ and impact ionization is not observed at this electric field even for a material thickness of $50 \mu \mathrm{m}$ (Juska et al. 1995). For holes, a poor mobility is observed, with a typical value of $\mu_{h} \sim 6 \mathrm{~cm}^{2} / \mathrm{sV}$. The density of traps in a regions between $0.3 \mathrm{eV}$ and $0.6 \mathrm{eV}$ adjacent to both the conduction and valence band edges is $\sim 5 \times 10^{16} \mathrm{~cm}^{-3} \mathrm{eV}^{-1}$ whilst the conduction and valence band tail cross sections are $s_{n}=s_{p} \sim 10^{-15} \mathrm{~cm}^{2}$ (Kleider et al. 1995). It was found that bimolecular recombination is the main mechanism for degradation of the electrical performance of this material, however charge collection efficiency values of up to $100 \%$ were observed even in quite thick samples $(50 \mu \mathrm{m})$ (Juska et al. 1999).

Recent progress in the development of direct deposition a-Si CMOS devices has proven the potential of this type of device as a cost-effective route for the fabrication of monolithic pixel detectors. Further studies are required to confirm the radiation hardness of these devices, which may offer a particular solution for pixellated tracking detectors located in the very harshest radiation environments. 


\section{Conclusions.}

At the present time $\mathrm{SiC}$ and $\mathrm{GaN}$ are the two main candidate materials that represent the most advanced alternatives to silicon for meeting the requirements of RD50 for radiation hard detectors. The ongoing development of $\mathrm{SiC}$ and $\mathrm{GaN}$ is being driven by the wider interest in these materials for use in high frequency electronics, optoelectronic, high temperature and high voltage device applications. The development of prototype radiation sensors fabricated from both $\mathrm{SiC}$ and $\mathrm{GaN}$ is well underway, and is particularly advanced in the case of SiC. Progress in these devices is generally still limited by the availability of high quality material that meets the specification required for radiation detector devices, rather than any serious processing or device technology problems.

For $\mathrm{SiC}$ radiation detectors only epitaxial material currently provides layers of suitable quality, in terms of low carrier concentration, long carrier lifetimes and mobilities, and the absence of charge trapping. However such layers are not yet available as SI free-standing films of suitable thickness, with a maximum thickness at present of approximately $50 \mu \mathrm{m}$. In order to achieve sufficient signal-noise ratio for MIP detection, it will be necessary to develop material approaching $300 \mu \mathrm{m}$ thickness for all but the most specific of applications. The quality of bulk $\mathrm{SiC}$ is currently inferior to that produced by epitaxy, and this material is less promising at the present time. However new developments in growth technologies continue at a rapid pace, and the availability of thicker SI $\mathrm{SiC}$ epitaxial layers with very low dislocation densities continues to improve. For GaN the situation is similar, with vapour phase epitaxy the preferred growth method for the production of thick layers. Currently SI GaN layers are only available on substrates, with thicknesses of $<10 \mu \mathrm{m}$. However various companies are developing HVPE technologies for growth of ultra-low dislocation density material, and are now starting to release commercially available free-standing thick material.

a-Si(H) is already finding application as a 'direct deposition' material suitable for fabrication of monolithic CMOS pixel detectors. First data from these devices shows a good sensitivity to particles, that needs further optimisation by increasing the $\mu \tau$ products of the charge carriers. For active pixel sensor devices, a-Si(H) offers an exciting new technology that has great promise.

The radiation hardness of these various materials is still an open question, and is the main theme of research which is currently being investigated within RD50. It is vital to show that the performance of these new materials at very high fluence are not only as good as that of silicon devices, but offer genuine performance advantages. As prototype detectors fabricated from these new materials become more easily available, we shall expect to see a wealth of new data that will answer the questions about radiation hardness and provide us with insights to the fundamental radiation interaction processes in these devices.

\section{Acknowledgements}

The authors are grateful to the many colleagues, both within RD50 and elsewhere, who contributed to this review paper through their discussions and comments, and by the supply of additional references and figures. 


\begin{tabular}{|c|c|c|c|c|c|c|c|c|c|c|}
\hline Property & Diamond & $\mathbf{S i}$ & a-Si(H) & $4 \mathrm{H}-\mathrm{SiC}$ & 6H-SiC & GaN & GaAs & $\operatorname{Cd}(Z n) T e$ & TIBr & $\mathrm{HgI}_{2}$ \\
\hline $\mathbf{Z}$ & 6 & 14 & 14 & $14 / 6$ & $14 / 6$ & $31 / 7$ & $31 / 33$ & $48 / 52$ & $81 / 35$ & $80 / 53$ \\
\hline$E_{g}[e V]$ & 5.5 & 1.12 & 1.7 & 3.3 & 3.03 & 3.39 & 1.4 & $1.4-1.6$ & 2.7 & 2.1 \\
\hline$\mu_{\mathrm{e}}\left[\mathrm{cm}^{2} / \mathrm{Vs}\right]$ & $\begin{array}{l}1800- \\
2200\end{array}$ & 1450 & $1-10$ & $\begin{array}{l}800- \\
1000\end{array}$ & 370 & 1000 & $=8500$ & 1000 & 40 & 100 \\
\hline$\mu_{\mathrm{h}}\left[\mathrm{cm}^{2} / \mathrm{Vs}\right]$ & $\begin{array}{l}1200- \\
1600\end{array}$ & 450 & $\begin{array}{l}0.01- \\
0.005\end{array}$ & $50-115$ & 50 & 30 & $=400$ & & 12 & 4 \\
\hline $\begin{array}{l}\text { Saturated electron } \\
\text { drift velocity }(\mathrm{cm} / \mathrm{s})\end{array}$ & $2.7 \times 10^{7}$ & $1.0 \times 10^{7}$ & & $2.0 \times 10^{7}$ & $2.0 \times 10^{7}$ & & $1.2 \times 10^{7}$ & & & \\
\hline e-h pair creation $[\mathrm{eV}]$ & 13 & 3.6 & $4-4.8$ & 7.8 & & 8.9 & 4.3 & $4.4-4.7$ & 5.9 & 4.2 \\
\hline eV/um for MIPs & 36 & 81 & & 51 & & & & & & \\
\hline Displacement [eV] & 43 & $13-20$ & & 21.8 & & $\begin{array}{l}\mathrm{Ga}-20 \\
\mathrm{~N}-10\end{array}$ & 10 & & & \\
\hline Density [g/cm3] & 3.5 & 2.3 & 2.3 & 3.2 & & 6.2 & 5.3 & $5.9-6.0$ & 7.5 & 6.4 \\
\hline$\varepsilon_{\mathbf{R}}$ & 5.5 & & & 9.7 & 10 & & & & & 8.8 \\
\hline $\begin{array}{l}\text { Breakdown voltage, } \\
{[\mathrm{MV} / \mathrm{cm}]}\end{array}$ & 10 & 0.5 & & $4^{1}$ & 2.4 & & $\sim 0.4$ & & & \\
\hline
\end{tabular}

Table 1: Material properties of major semiconductors for use as ionising radiation detectors

${ }^{1}$ Other data has $2.2 \mathrm{MV} / \mathrm{cm}$ 
Figures

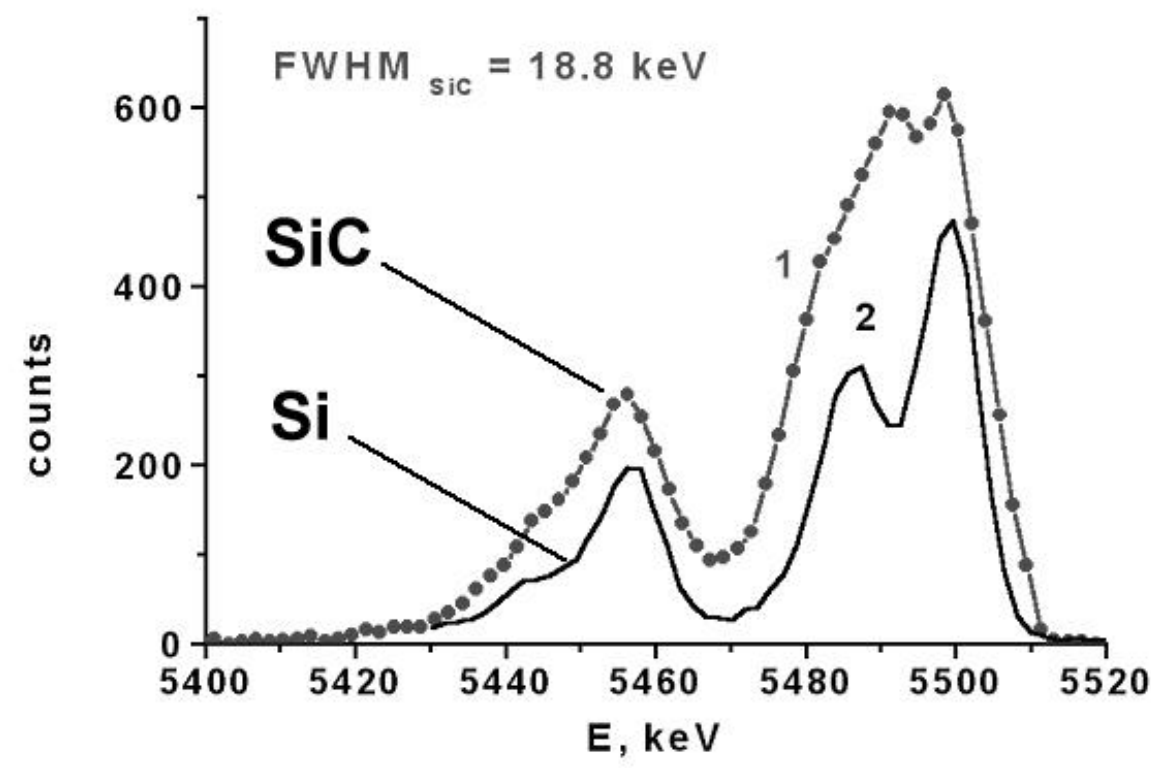

Figure 1: Alpha particle pulse height spectra showing fine structure from ${ }^{241} \mathrm{Am}$, from $4 \mathrm{H}-\mathrm{SiC}$ and Si detectors (Ivanov et al. 2005).

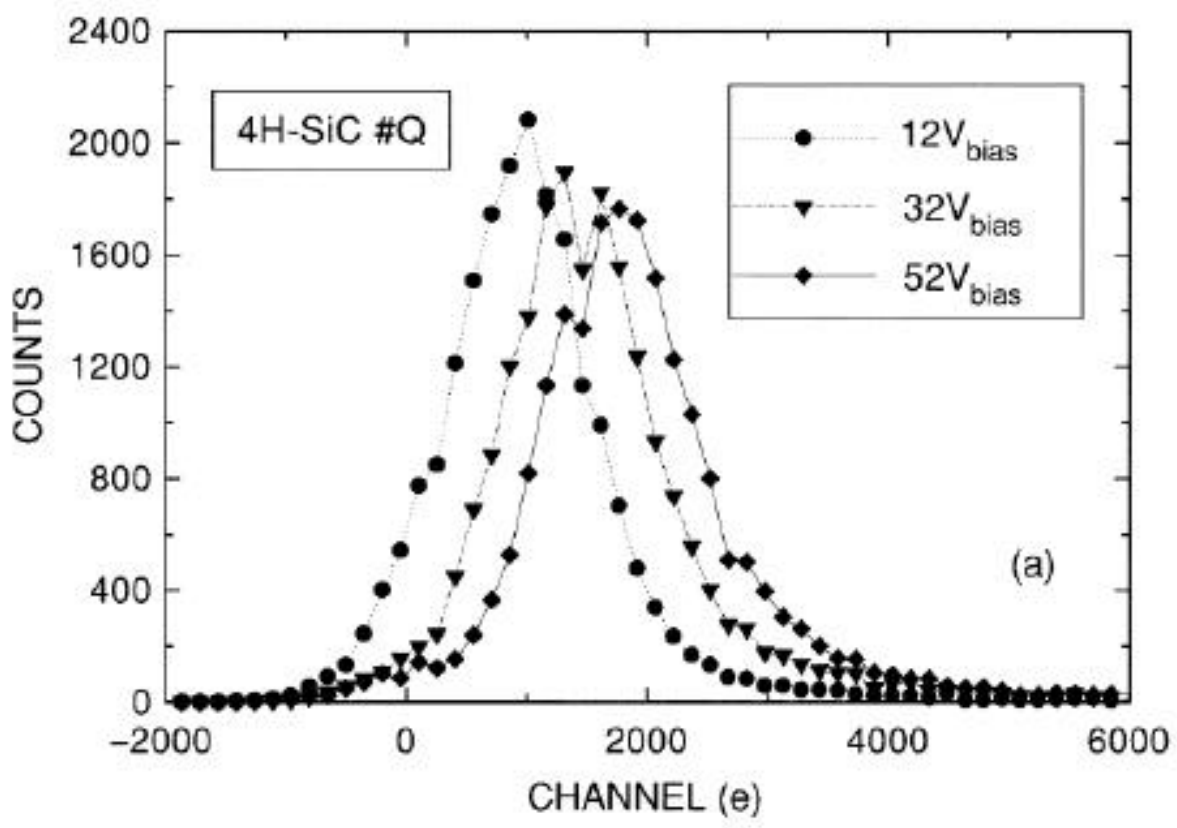

Figure 2: Landau spectrum acquired from a ${ }^{90} \mathrm{Sr}$ source from a $40 \mu \mathrm{m}$ thick epitaxial $4 \mathrm{H}-\mathrm{SiC}$ detector (Nava et al. 2004). 


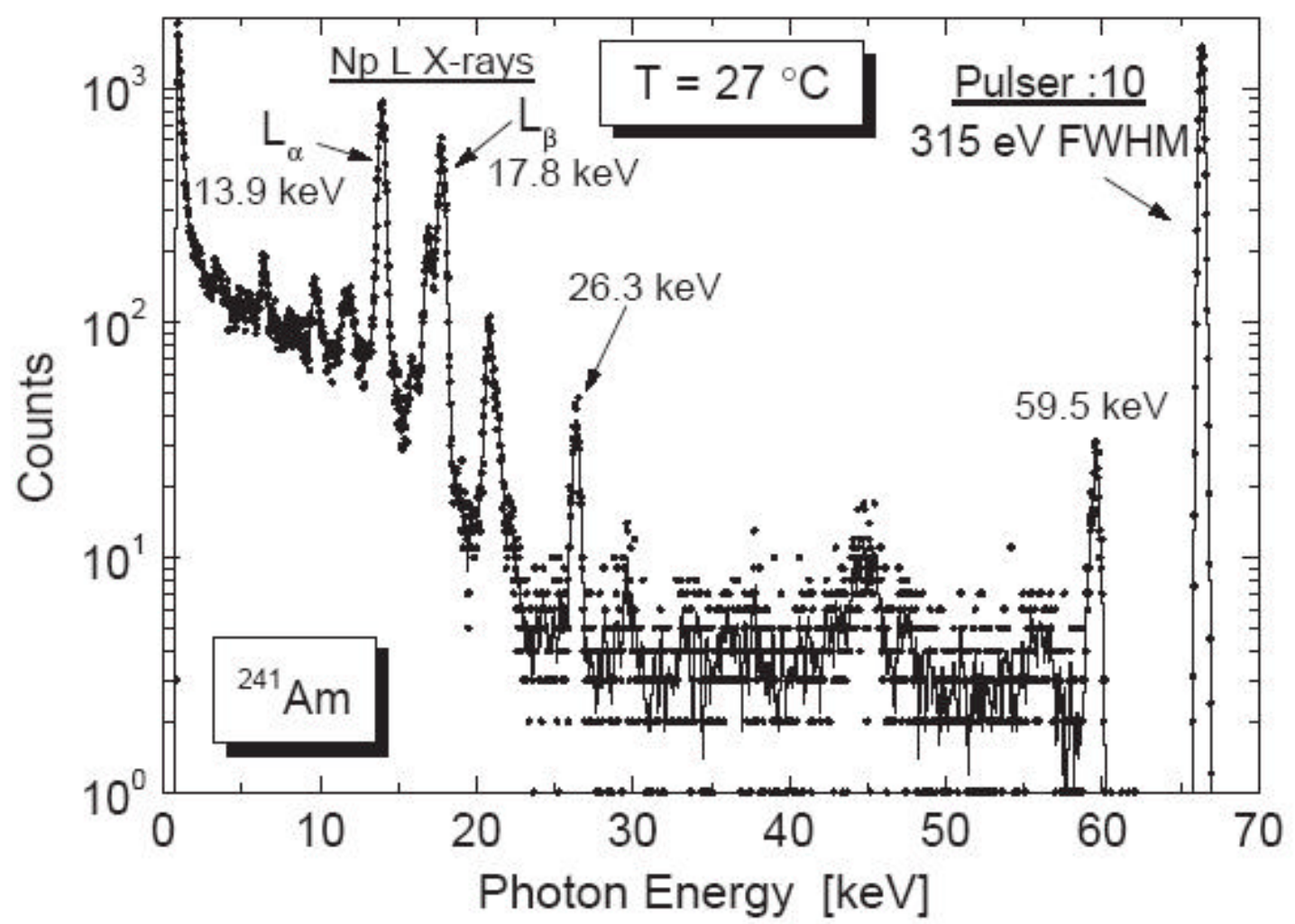

Figure 3: X-ray and gamma ray response from ${ }^{241} \mathrm{Am}$, recorded by an epitaxial $4 \mathrm{H}-\mathrm{SiC}$ detector $\mathrm{SiC}$ detector of $0.03 \mathrm{~mm}^{2}$ at $27^{\circ} \mathrm{C}$. The shaping time is $12 \mu \mathrm{s}$. The equivalent noise energy is 315 eV FWHM (Bertuccio et al. 2004). 


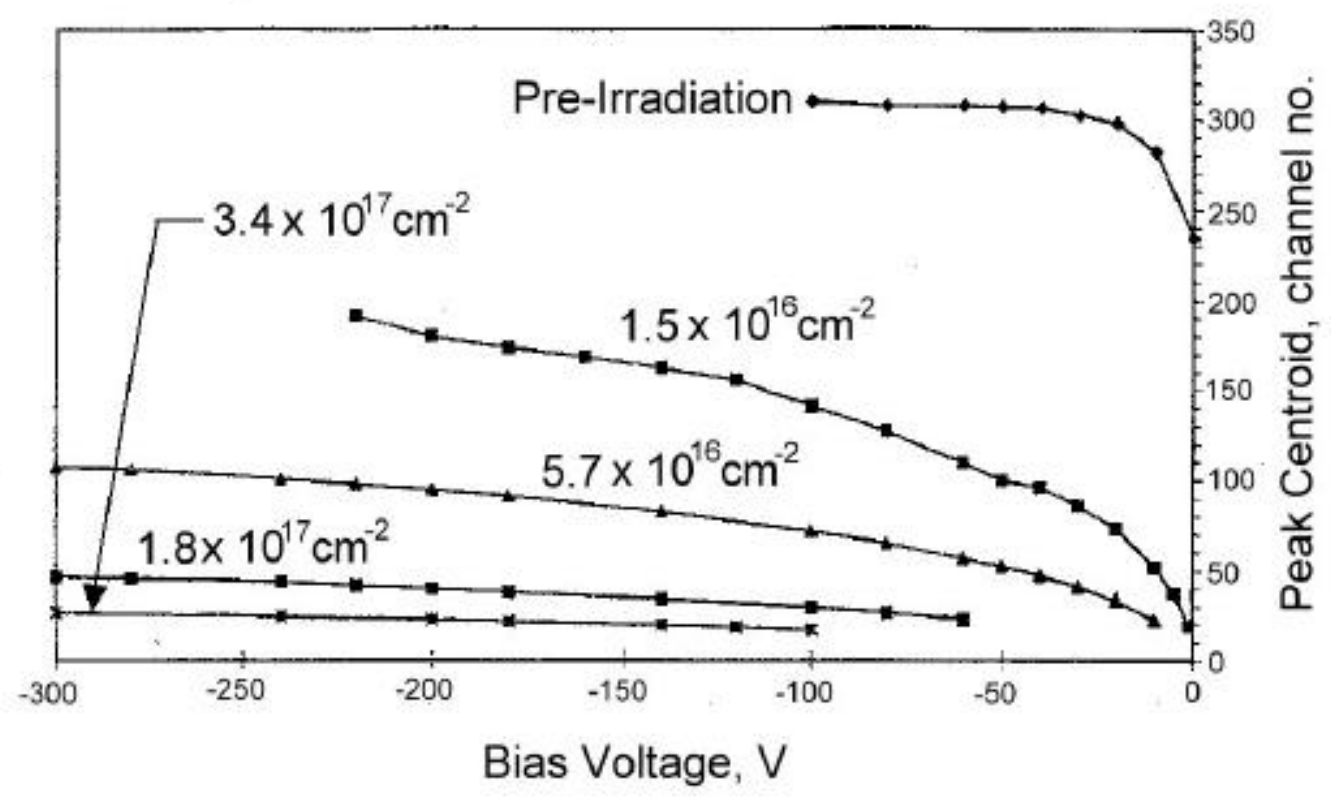

Figure 4: Alpha particle peak centroid versus bias voltage for a SiC Schottky diode before and after exposure to a range of thermal neutron fluences (Seshadri et al. 1999).

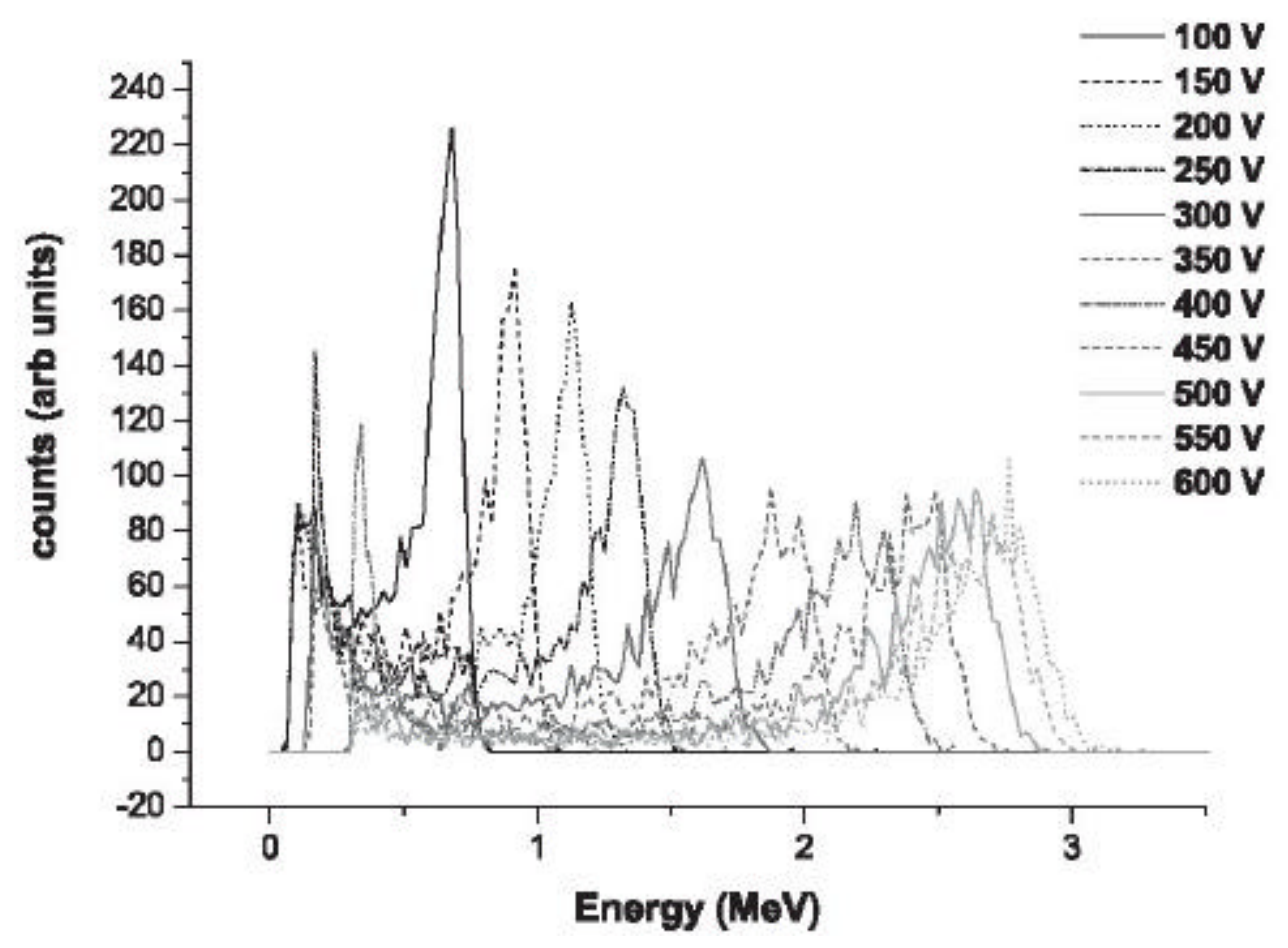

Figure 5: Alpha particle spectrum acquired from a bulk $\mathrm{SiC}$ radiation detector, after irradiation with $10^{13}$ pions $/ \mathrm{cm}^{2}$ (Cunningham et al. 2003). 


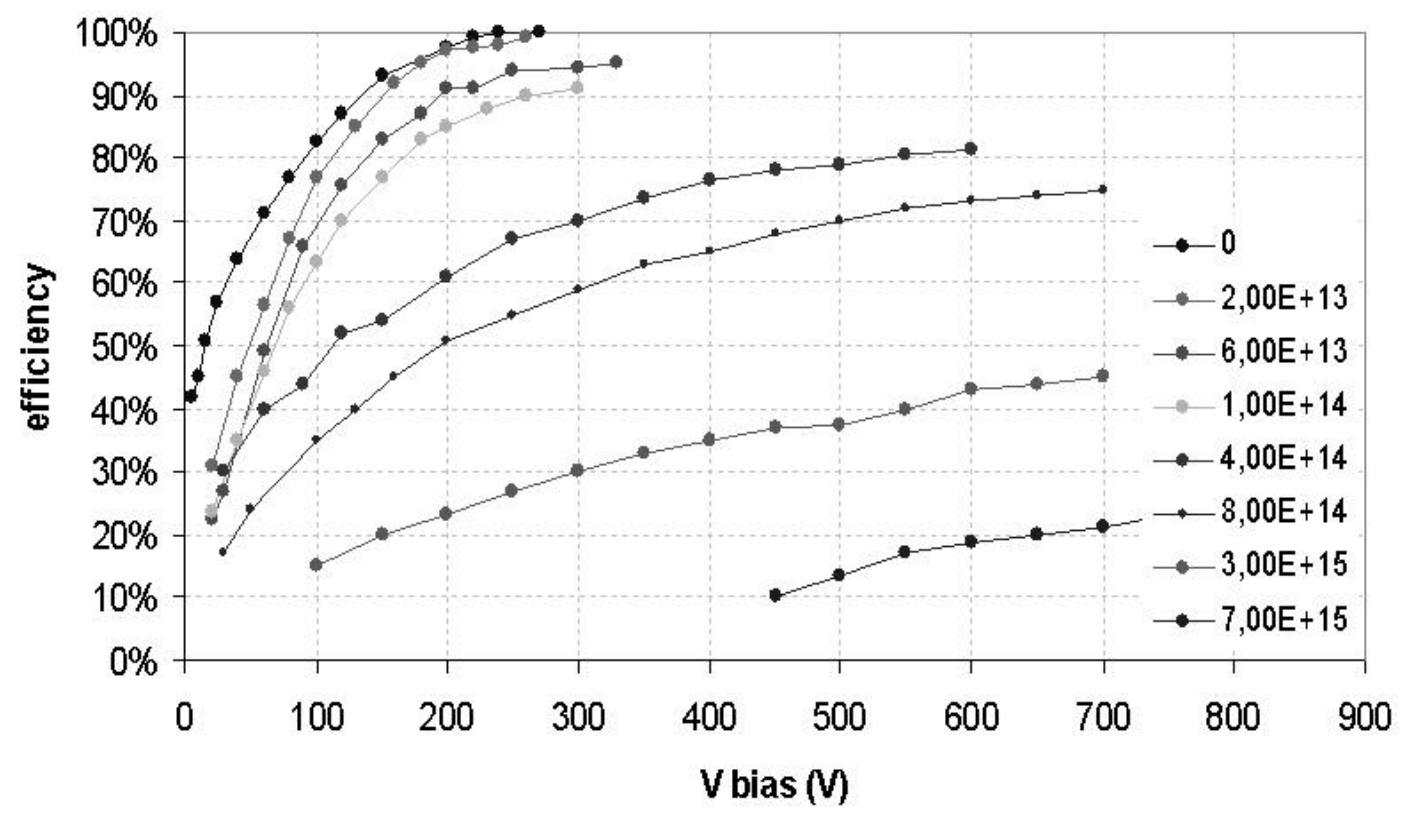

Figure 6: alpha particle CCE for a $21 \mu \mathrm{m}$ thick epitaxial $4 \mathrm{H}-\mathrm{SiC}$ detector, as a function of neutron fluence (Sciortino et al. 2005).

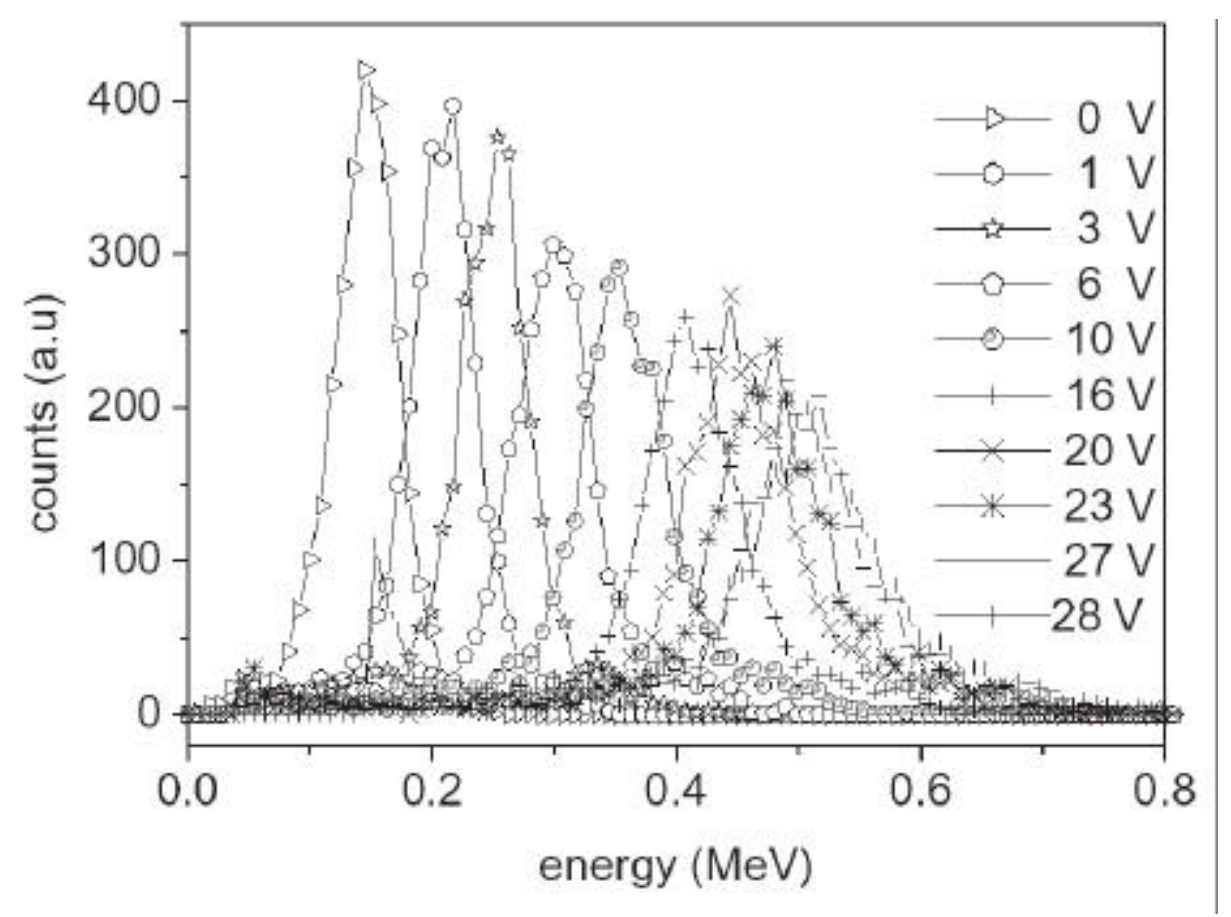

Figure 7: alpha particle pulse height spectra obtained from a $2 \mu \mathrm{m}$ thick SI GaN Schottky diode (Vaitkus et al. 2003). 


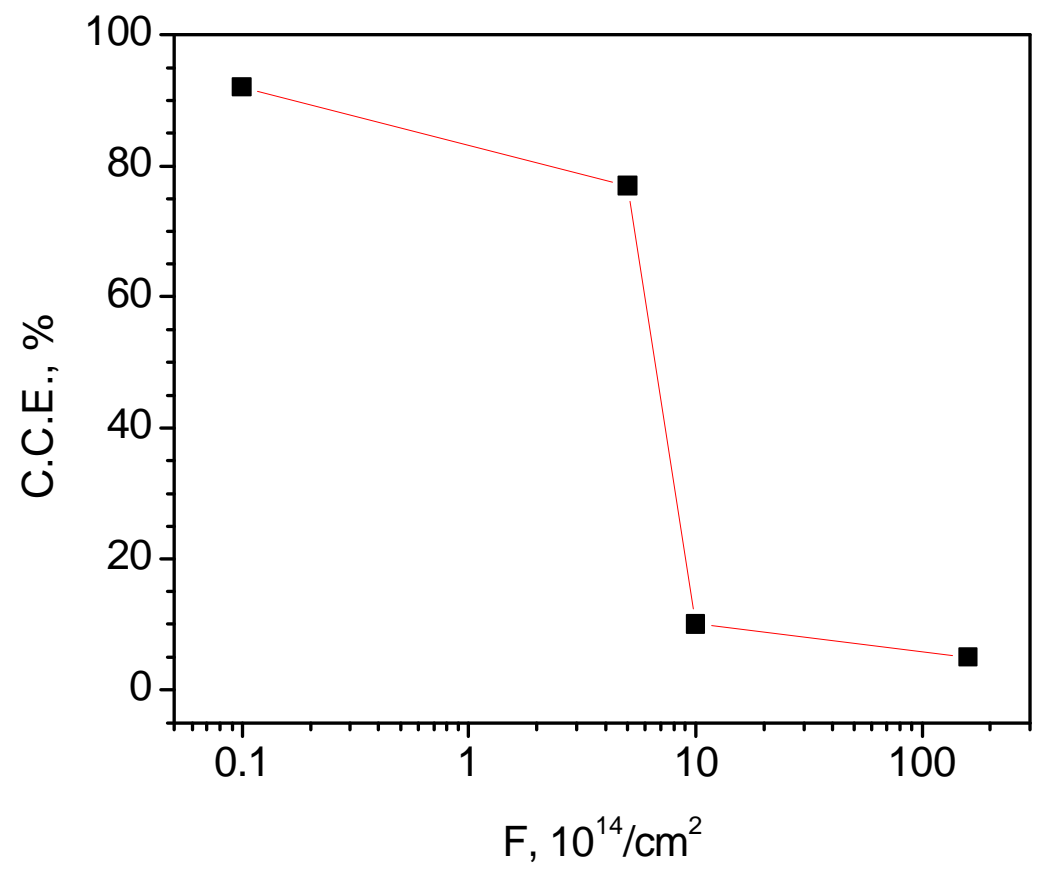

Figure 8:CCE measured from a neutron-irradiated SI GaN detector, of $2 \mu \mathrm{m}$ thickness (Vaitkus 2005).

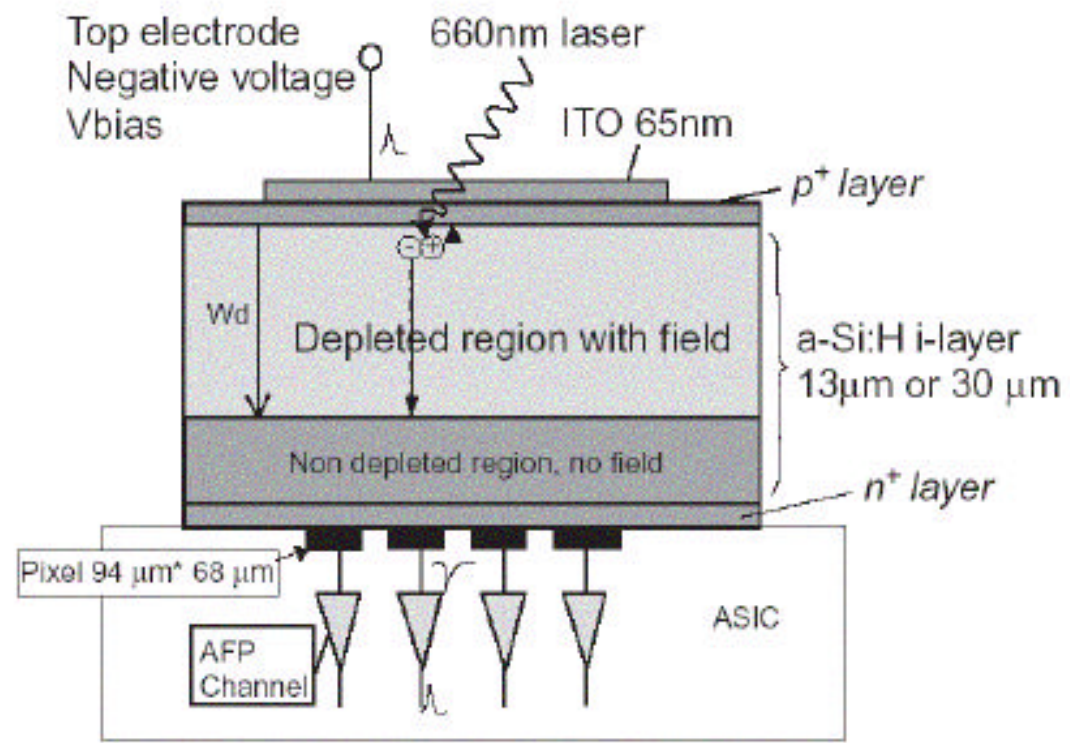

Figure 9: Monolithic pixel detector fabricated from direct deposition of a-Si:H onto a CMOS ASIC (Despeisse et al. 2004). 


\section{References}

Adam, W., et al. (2003). "Status of the R\&D activity on diamond particle detectors." Nuclear Instruments and Methods in Physics Research Section A 511(1/2): 124-131.

Aizenshtadt, G. I., et al. (2000). "Ionizing-radiation detectors based on GaAs with deep centers." Nuclear Instruments and Methods in Physics Research Section A 448(1-2): 188-191.

Alvarez, A., et al. (1995). "Selected Properties of Hydrogenated Amorphous Silicon and SiliconCarbon Alloys." Solid State Phenomena(44//6/1): 3.

Anelli, G., et al. (2004). "A new concept of monolithic silicon pixel detectors: hydrogenated amorphous silicon on ASIC." Nuclear Instruments and Methods in Physics Research Section A 518(1-2): 366-372.

Augustine, G., et al. (1997). Physical Vapor Transport Growth and Properties of SiC Monocrystals of 4H Polytype. Fundamental Questions and Applications of SiC (Part I). W. J. Choyke and H. Matsunami, Academic Verlag Gmbh. 202; Number 1: 137-148.

Barrett, D. L., et al. (1993). "Growth of large SiC single crystals." Journal of Crystal Growth 128(1//4-1): 358 .

Bates, R., et al. (1998). "Gallium arsenide pixel detectors." Nuclear Instruments and Methods in Physics Research Section A 410(1): 6-11.

Bertuccio, G., et al. (2003). "Study of silicon carbide for X-ray detection and spectroscopy." Ieee Transactions on Nuclear Science 50(1): 175-185.

Bertuccio, G., et al. (2004). "Silicon carbide for high resolution X-ray detectors operating up to 100degreeC." Nuclear Instruments and Methods in Physics Research Section A 522(3): 413-419.

Bertuccio, G., et al. (2003). "Noise Analysis of Gallium Arsenide Pixel X-Ray Detectors Coupled to Ultra-Low Noise Electronics." Ieee Transactions on Nuclear Science 50(3; Part 4): 723728.

Bickermann, M., et al. (2001). "Incorporation of boron and vanadium during PVT growth of 6HSiC crystals." Journal of Crystal Growth 233(1-2): 211-218.

Bickermann, M., et al. (2001). "On the preparation of semi-insulating SiC bulk crystals by the PVT technique." Applied Surface Science 184(1-4): 84-89.

Bruzzi, M. (2004). "Material engineering for the development of ultra-radiation hard semiconductor detectors." Nuclear Instruments and Methods in Physics Research Section A 518(1-2): 336-337.

Bruzzi, M., et al. (2003). "Characterisation of epitaxial SiC Schottky barriers as particle detectors." Diamond and Related Materials 12(3-7): 1205-1208.

Bruzzi, M., et al. (2001). "High quality SiC applications in radiation dosimetry." Applied Surface Science 184(1-4): 425-430.

Bruzzi, M., et al. (2001). "Characterisation of silicon carbide detectors response to electron and photon irradiation." Diamond and Related Materials 10(3-7): 657-661.

Castaldini, A., et al. (2002). "Deep levels in silicon carbide Schottky diodes." Applied Surface Science 187(3-4): 248-252.

Cunningham, W., et al. (2003). "Performance of irradiated bulk SiC detectors." Nuclear Instruments and Methods in Physics Research A 509: 127-131. 
Cunningham, W., et al. (2003). "Performance of irradiated bulk SiC detectors." Nuclear Instruments and Methods in Physics Research Section A 509(1-3): 127-131.

Dalibor, T., et al. (1997). "Deep Defect Centers in Silicon Carbide Monitored with Deep Level Transient Spectroscopy." Physica Status Solidi a Applied Research 162: 199-226.

Despeisse, M., et al. (2004). "Characterization of 13 and 30mm thick hydrogenated amorphous silicon diodes deposited over CMOS integrated circuits for particle detection application." Nuclear Instruments and Methods in Physics Research Section A 518(1-2): 357-361.

Dhar, S., et al. (2000). "Low field electron mobility in 6H-SiC." Journal of Applied Physics 88(11): 6519-6525.

Filip, O., et al. (2004). "Micropipe healing in $\mathrm{SiC}$ wafers by liquid-phase epitaxy in Si-Ge melts." Journal of Crystal Growth 271(1-2): 142-150.

Freitas, J. A., et al. (2002). "Donors in hydride-vapor-phase epitaxial GaN." Journal of Crystal Growth 246(3-4): 307-314.

Fujihira, K., et al. (2003). "Growth and characterization of 4H-SiC in vertical hot-wall chemical vapor deposition." Journal of Crystal Growth 255(1/2): 136-144.

Galeckas, A., et al. (2002). "Recombination-enhanced extension of stacking faults in 4H-SiC p-i-n diodes under forward bias." Applied Physics Letters 81(5): 883-885.

Gaubas, E., et al. (2005). "Defect attributed variations of the photoluminescence and photoconductivity in the HVPE and MOCVD as-grown and irradiated GaN structures." To be published in Nuclear Instruments and Methods in Physics Research A.

Giocondi, J., et al. (1997). "An atomic force microscopy study of super-dislocation/micropipe complexes on the $6 \mathrm{H}-\mathrm{SiC}(0001)$ growth surface." Journal of Crystal Growth 181(4): 351362.

Hobgood, H. M., et al. (1994). "Large diameter 6H-SiC for microwave device applications." Journal of Crystal Growth 137(1//2): 181.

Holmes, D. E., et al. (1988). "Dislocation reduction in large-diameter LEC GaAs growth : I. Low gradient growth and indium doping." Journal of Crystal Growth 91(4): 557-566.

Ionascut-Nedelcescu, A., et al. (2002). "Radiation Hardness of Gallium Nitride." Ieee Transactions on Nuclear Science 49(6; Part 1): 2733-2738.

Ivanov, A. M., et al. (2005). "High energy resolution detectors based on 4H-SiC." To be published in proceedings of the ECSCRM-2004 conference, Bologna.

Jackson, W. B., et al. (1988). Kinetics of carrier-induced metastable defect formation in hydrogenated amorphous silicon, World Scientific Publishing Company.

Jacobson, H., et al. (2004). "Properties and origins of different stacking faults that cause degradation in SiC PiN diodes." Journal of Applied Physics 95(3): 1485-1488.

Jenny, J. R., et al. (1996). "Deep level transient spectroscopic and Hall effect investigation of the position of the vanadium acceptor level in $4 \mathrm{H}$ and $6 \mathrm{H} \mathrm{SiC."} \mathrm{Applied} \mathrm{Physics} \mathrm{Letters}$ 68(14): 1963-1965.

Juska, G., et al. (1995). "Properties of Hot Carriers in a-Si:H in Comparison to a-Se." Solid State Phenomena 44-46: 551-558.

Juska, G., et al. (1995). "Hot Electrons in Amorphous Silicon." Physical Review Letters 75(16): 2984.

Juska, G., et al. (1999). "Ultrafast Charge Carrier Recombination in a-Si:H and æc-Si:H." Physica Status Solidi a Applied Research 171(2): 539-548. 
Kalinina, E. V., et al. (2004). "Optical and Electrical Properties of 4H-SiC Irradiated with Fast Neutrons and High-Energy Heavy Ions." Semiconductors 38(10): 1187-1191.

Kelly, M. K., et al. (1999). "Large Free-Standing GaN Substrates by Hydride Vapor Phase Epitaxy and Laser-lnduced Liftoff." Japanese Journal of Applied Physics Part 2 Letters 38(3a): L 217-L 219.

Kishimoto, N., et al. (1998). "Radiation resistance of amorphous silicon in optoelectric properties under proton bombardment." Journal of Nuclear Materials 258/263(B): 1908-1913.

Kleider, J. P., et al. (1995). "Applications of the Modulated Photocurrent Technique to the Determination of Gap States Characteristics in Hydrogenated Amorphous Silicon." Solid State Phenomena(44-46): 597-646.

Lebedev, A. A., et al. (2004). "Radiation resistance of SiC and nuclear-radiation detectors based on SiC films." Semiconductors 38(2): 125-147.

Lindstrom, G., et al. (2001). "Developments for radiation hard silicon detectors by defect engineering-results by the CERN RD48 (ROSE) Collaboration." Nuclear Instruments and Methods in Physics Research Section A 465(1): 60-69.

Luukka, P. (2004). "Status of defect engineering activity of the RD50 collaboration." Nuclear Instruments and Methods in Physics Research Section A 530(1-2): 152-157.

$\mathrm{Ma}, \mathrm{X}$., et al. (2002). "Nondestructive defect delineation in SiC wafers based on an optical stress technique." Applied Physics Letters 80(18): 3298-3300.

Mainwood, A. (2000). "Recent developments of diamond detectors for particles and UV radiation." Semiconductor Science and Technology 15(9): R55.

Metzger, S., et al. (2002). "Silicon Carbide Radiation Detector for Harsh Environments." Ieee Transactions on Nuclear Science Ns 49(3; Part 3): 1351-1355.

Milita, S., et al. (1999). "Coherent X-ray imaging investigation of macrodefects and micropipes on SiC." Materials Science and Engineering 61-62(1): 63 - 67.

Mitchel, W. C., et al. (1999). "Fermi level control and deep levels in semi-insulating 4H-SiC." Journal of Applied Physics 86(9): 5040-5044.

Moll, M. (2003). "Development of radiation hard sensors for very high luminosity collidersCERN-RD50 project." Nuclear Instruments and Methods in Physics Research Section A 511(1/2): 97-105.

Muzykov, P. G., et al. (2003). "High Resistivity Measurement of SiC Wafers Using Different Techniques." Journal of Electronic Materials 32(6): 505-510.

Nakamura, S., et al. (2000). Introduction to nitride semiconductor blue lasers and light emitting diodes. London, Taylor and Francis.

Nava, F., et al. (2004). "Minimum Ionizing and Alpha Particles Detectors Based on Epitaxial Semiconductor Silicon Carbide." Ieee Transactions on Nuclear Science 51 (1; Part 2): 238244.

Nava, F., et al. (1999). "Epitaxial silicon carbide charge particle detectors." Nuclear Instruments and Methods in Physics Research Section A 437(2-3): 354-358.

Nava, F., et al. (2003). "Investigation of Ni/4H-SiC diodes as radiation detectors with low doped ntype 4H-SiC epilayers." Nuclear Instruments and Methods in Physics Research Section A 510(3): 273-280.

Owens, A., et al. (1999). "X-ray response of epitaxial GaAs." Journal of Applied Physics 85(11): $7522-7527$. 
Pau, J. L., et al. (2004). "Response of ultra-low dislocation density GaN photodetectors in the nearand vacuum-ultraviolet." Journal of Applied Physics 95(12): 8275-8279.

Porowski, S., et al. (1997). "Thermodynamical properties of III-V nitrides and crystal growth of GaN at high N 2 pressure." Journal of Crystal Growth 178(1/2): 174-188.

Rahman, M., et al. (2003). "Super-Radiation Hard Particle Tracking at the CERN SLHC." Ieee Transactions on Nuclear Science 50(6; Part 1): 1797-1804.

Reimer, J. A., et al. (1988). Structural heterogeneities in device-quality amporphous hydrogenated semiconductors, World Scientific Publishing Company.

Rogalla, M., et al. (1999). "Particle detectors based on semi-insulating silicon carbide." Nuclear Physics B 78: 516-520.

Ruddy, E. H., et al. (1998). "Development of a Silicon Carbide Radiation Detector." Ieee Transactions on Nuclear Science Ns 45(3; Number 1): 536-541.

Saravanan, S., et al. (2000). "Impurity reduction and crystalline quality improvement due to isovalent doping (In) in GaAs epilayers on Si substrate by chemical beam epitaxy." Journal of Crystal Growth 209(4): 621-624.

Schadt, M., et al. (1994). "Anisotropy of the electron Hall mobility in 4H, 6H, and 15R silicon carbide." Applied Physics Letters 65(24): 3120.

Schmidt, U. I., et al. (1995). "Ellipsometric Studies of a-Si:H Film Growth, Density and Microstructure." Solid State Phenomena(44//6/1): 195.

Schneider, J., et al. (1990). "Infrared spectra and electron spin resonance of vanadium deep level impurities in silicon carbide." Applied Physics Letters 56: 1184-1186.

Schneider, U., et al. (1988). Metastable defects in hydrogenated amorphous silicon (a-Si:H) produced by electron irradiation, World Scientific Publishing Company.

Sciortino, S., et al. (2005). "Effect of heavy proton and neutron irradiations on epitaxial 4H-SiC Schottky diodes." To be published in Nuclear Instruments and Methods in Physics Research A, proceedings of the RESMDD 04 conference, Florence.

Sellin, P. J. (2003). "Recent advances in compound semiconductor radiation detectors." Nuclear Instruments and Methods in Physics Research Section A 513(1-2): 332-339.

Sellin, P. J., et al. (2004). "Ion beam induced charge imaging of epitaxial GaN detectors." Nuclear Instruments and Methods in Physics Research Section A 531(1-2): 82-86.

Seshadri, S., et al. (1999). "Demonstration of an SiC Neutron Detector for High-Radiation Environments." Ieee Transactions on Electron Devices 46(3): 567-571.

Shur, M. S., et al. (2004). UV Solid State Light Emitters and Detectors. NATO Science Series. M. S. Shur and A. Zukauskas. Dordrecht, Kluwer Academic Publishers. 144.

Street, R. A., et al. (1996). "Large area amorphous silicon x-ray imagers." Nuclear Instruments and Methods in Physics Research Section A 380: 450-454.

Vaitkus, J. (2005). "To be confirmed." To be published in Nuclear Instruments and Methods in Physics Research A, proceedings of the RESMDD 04 conference, Florence.

Vaitkus, J., et al. (2003). "Semi-insulating GaN and its evaluation for alpha particle detection." Nuclear Instruments and Methods in Physics Research A: 60-64.

Vaitkus, J., et al. (2005). A new radiation hard semiconductor - semi-insulating GaN: Photoelectric properties. ICPS-27, AIP.

Vaitkus, J., et al. (2003). "Space charge effects, carrier capture transient behaviour and a particle detection in semi-insulating GaN." Nuclear Instruments and Methods in Physics Research Section A 514(1-3): 141-145. 
Verzellesi, G., et al. (2002). "Investigation on the charge collection properties of a $4 \mathrm{H}-\mathrm{SiC}$ Schottky diode detector." Nuclear Instruments and Methods in Physics Research Section A 476(3): 717-721.

Wang, T., et al. (2000). "Influence of buffer layer and growth temperature on the properties of an undoped $\mathrm{GaN}$ layer grown on sapphire substrate by metalorganic chemical vapor deposition." Applied Physics Letters 76(16): 2220-2222.

Wyrsch, N., et al. (1995). "Recent Progress in the Interpretation of a-Si:H Transport Properties: Lifetimes, Mobilities and Mobility-Lifetime Products." Solid State Phenomena(44-46): 525-534.

$\mathrm{Xu}, \mathrm{X}$., et al. (2002). "Growth and characterization of low defect $\mathrm{GaN}$ by hydride vapor phase epitaxy." Journal of Crystal Growth 246(3-4): 223-229.

Yang, Q., et al. (2003). "Observation of a hydrogenic donor in the luminescence of electronirradiated GaN." Applied Physics Letters 82(18): 3002-3004. 